\title{
High-speed tapping-mode atomic force microscopy using a $Q$-controlled regular cantilever acting as the actuator: Proof-of-principle experiments
}

\author{
M. Balantekin, ${ }^{1, a)}$ S. Satır, ${ }^{2}$ D. Torello, ${ }^{2}$ and F. L. Değertekin ${ }^{2}$ \\ ${ }^{1}$ Electrical and Electronics Engineering, İmir Institute of Technology, Urla, Izmir 35430, Turkey \\ ${ }^{2}$ Woodruff School of Mechanical Engineering, Georgia Institute of Technology, Atlanta, \\ Georgia 30332-0405, USA
}

(Received 6 September 2014; accepted 24 November 2014; published online 10 December 2014)

\begin{abstract}
We present the proof-of-principle experiments of a high-speed actuation method to be used in tappingmode atomic force microscopes (AFM). In this method, we do not employ a piezotube actuator to move the tip or the sample as in conventional AFM systems, but, we utilize a $Q$-controlled eigenmode of a cantilever to perform the fast actuation. We show that the actuation speed can be increased even with a regular cantilever. ( 2014 AIP Publishing LLC. [http://dx.doi.org/10.1063/1.4903469]
\end{abstract}

\section{INTRODUCTION}

The atomic force microscope (AFM) has enabled highresolution imaging, manipulation, and characterization of nanoscale structures, devices, and systems. However, the imaging speed of dynamic AFM systems is not satisfactory for some applications. In a dynamic AFM system, mechanical and electronic components, i.e., the actuator, the cantilever probe, the X-Y scanner, and the feedback controller, all affect the scan speed. In recent studies, sensor and actuator sizes have been minimized ${ }^{1-6}$ special probes with integrated actuators were designed, ${ }^{7-13}$ and fast scanners were developed. ${ }^{14-18}$ In fact, the bandwidth of the actuator and the transient response of the probe are the main factors that limit the scan speed.

We know that the probe and actuator sizes must be minimized to have fast sensing and actuation. We also know that the smallest component in an AFM system is the cantilever probe. Therefore if we can manage to use this smallest component for both sensing and actuation, then we could achieve higher scan rates. Recently, an actuation method was developed for this purpose. ${ }^{19}$ This actuation method can be effectively utilized in both the amplitude modulation (AM) AFM, also called tapping-mode, and the frequency modulation (FM) AFM, also called noncontact-mode. It was shown theoretically that the imaging speed can be increased by about 2 orders of magnitude compared to conventional dynamic AFM imaging. ${ }^{19}$

In this paper, we present the proof-of-principle experiments of this method in tapping-mode. The operating principle of the method is, briefly, to adjust the oscillation amplitude of the fundamental eigenmode to keep the higher eigenmode oscillation amplitude at a constant level. In other words, instead of using a Z-piezo for actuation as in conventional AFM systems, the fundamental eigenmode oscillation amplitude is adjusted to perform the actuation. It is known that the Z-piezo in the commercial AFM systems is the main factor limiting the system bandwidth and hence the scan speed due to its low resonant frequency. On the other hand, a typical tapping-

\footnotetext{
a) Author to whom correspondence should be addressed. Electronic mail:
} mujdatbalantekin@iyte.edu.tr mode cantilever has a resonant frequency on the order of a few hundred kHz. Consequently, a faster transient response is expected to the topographical variations if a cantilever is used as an actuator. However, a typical tapping-mode cantilever also has a high $Q$-factor in air. We solve this problem by applying the $Q$-control method ${ }^{20-25}$ to reduce the artificial $Q$-factor of the fundamental eigenmode. Hence the oscillation amplitude of fundamental eigenmode will reach the steady-state faster.

\section{EXPERIMENTS}

The method of high-speed tapping-mode imaging is implemented as shown in Fig. 1. The photodiode signal is the input of AFM controller which provides both $\mathrm{X}$-Y scan and Z actuation in the conventional system. The photodiode signal is connected to two analog band-pass filters which have center frequencies at the resonance frequencies of fundamental $\left(f_{1}\right)$ and higher $\left(f_{2}\right)$ eigenmodes. The outputs of the filters are connected to two amplitude detectors. Since the higher eigenmode oscillation is used as a sensing signal, we use its amplitude in a PI control unit. The PI controller output controls the variable gain amplifier (VGA) such that the fundamental eigenmode excitation $\left(f_{1}\right)$ is decreased if there is an increase in the surface topography and increased if there is a decrease in the surface topography. The $Q$-control is implemented by a cascaded variable gain $(\mathrm{G})$ and phase $(\phi)$ blocks. The output of the $Q$-control circuit, the VGA output, and the high frequency excitation $\left(f_{2}\right)$ signal are all added to obtain the signal applied to the tapping piezo which excites the cantilever.

The experiments were performed on the Veeco's Dimension 3100 AFM. The Signal Access Module was used to obtain the photodiode signal and to apply the tapping piezo signal (see Fig. 2). The additional system electronics were implemented with off-the-shelf components, the gain, phase, and set-point adjustments were performed with trimmer potentiometers. A peak detector is better choice for the amplitude detection, ${ }^{26}$ but, for simplicity we used a simple envelope detector. We used Analog Devices' AD603 evaluation kit as the VGA. The cantilever that we used is HA-NC etalon cantilever from NT-MDT that has fundamental and second eigenmode resonances of $f_{1}=113 \mathrm{kHz}$ and $f_{2}=725 \mathrm{kHz}$, respectively. In 


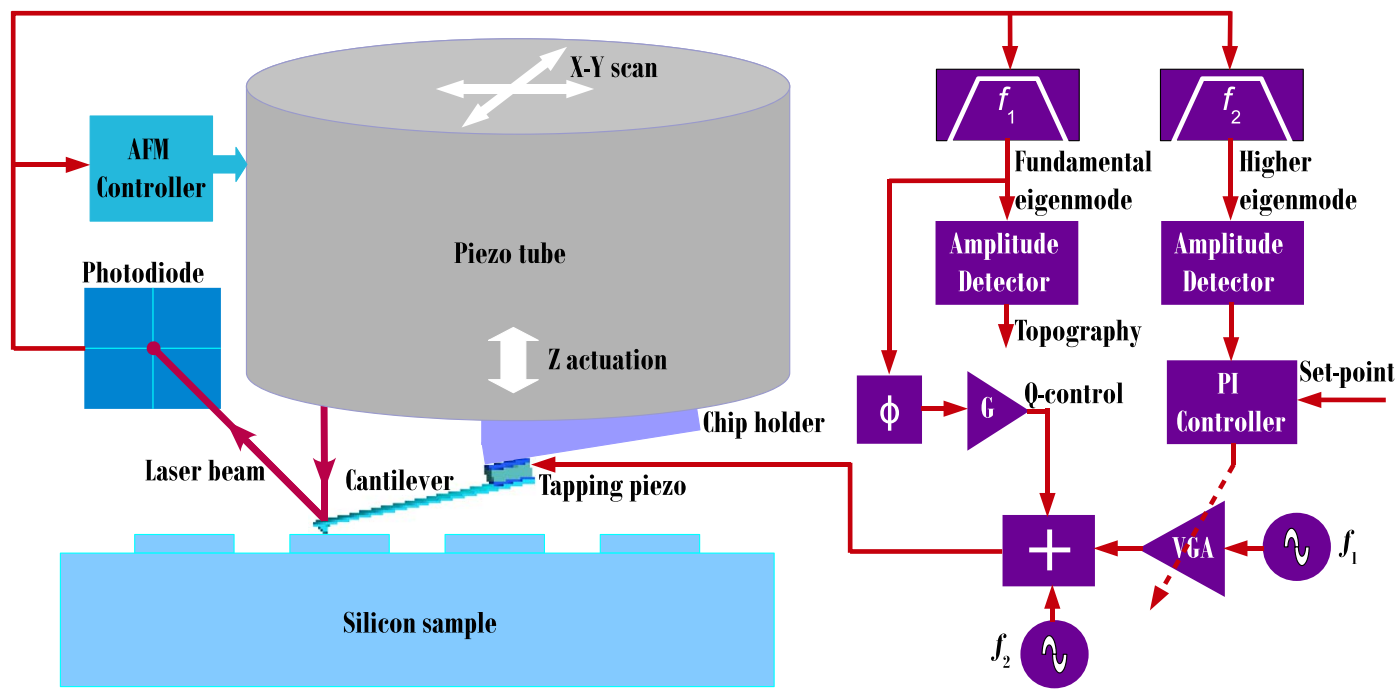

FIG. 1. Experimental setup. The photodiode signal is filtered by two band-pass filters at the resonance frequencies of fundamental and higher eigenmodes. The $Q$-control signal is obtained by a variable phase $(\phi)$ and gain (G) blocks. The variable gain amplifier (VGA) is controlled by the higher eigenmode amplitude through PI controller. $f_{1}$ and $f_{2}$ are the signal generator frequencies at the fundamental and higher eigenmode resonances.

the imaging experiments, we used a calibration sample shown in Fig. 3(a), a silicon grating with $4 \mu \mathrm{m}$ period and a $25 \mathrm{~nm}$ step height, from Nanoscience Instruments.

\section{RESULTS AND DISCUSSION}

The topography images obtained by conventional tapping-mode system at $1 \mathrm{~Hz}$ and $10 \mathrm{~Hz}$ scan rates are given in Figs. 3(b) and 3(c). In this case, the tapping piezo signal is comprised of only the fundamental eigenmode excitation. The free oscillation amplitude and set-point are approximately $30 \mathrm{~nm}$ and $70 \%$, respectively. The $\mathrm{Z}$ actuation is performed by the piezo tube. The gain of internal AFM controller is set to highest stable gain. We see that the image gets heavily distorted as the scan rate is increased from $1 \mathrm{~Hz}$ to $10 \mathrm{~Hz}$. In addition, the surface feature is seen to be moved up slightly and the topography range is changed by about $13 \%$.
Figures 3(d) and 3(e) show the amplitude images at $0.5 \mathrm{~Hz}$ and $10 \mathrm{~Hz}$ scan rates obtained by the proposed fast actuation method. The AFM controller only performs the $\mathrm{X}$-Y scan and the Z-actuation is disabled by setting the internal controller gain to zero. The inverse of the output voltage of the amplitude detector that measures the fundamental eigenmode oscillation amplitude must be proportional to the topography. Since the internal feedback controller gain is set to zero, the amplitude subtracted by the set-point (the error signal) must also be proportional to the sample topography. We utilized the latter option since we could not map the external fundamental amplitude signal. The free oscillation amplitude of the second eigenmode is about $10 \mathrm{~nm}$, and the oscillation amplitude of fundamental eigenmode can be changed roughly between $100 \mathrm{~nm}$ and $200 \mathrm{~nm}$. In Fig. 2, we see the tapping piezo signal when the VGA gain is high and so is the fundamental eigenmode oscillation amplitude, i.e., the tip is

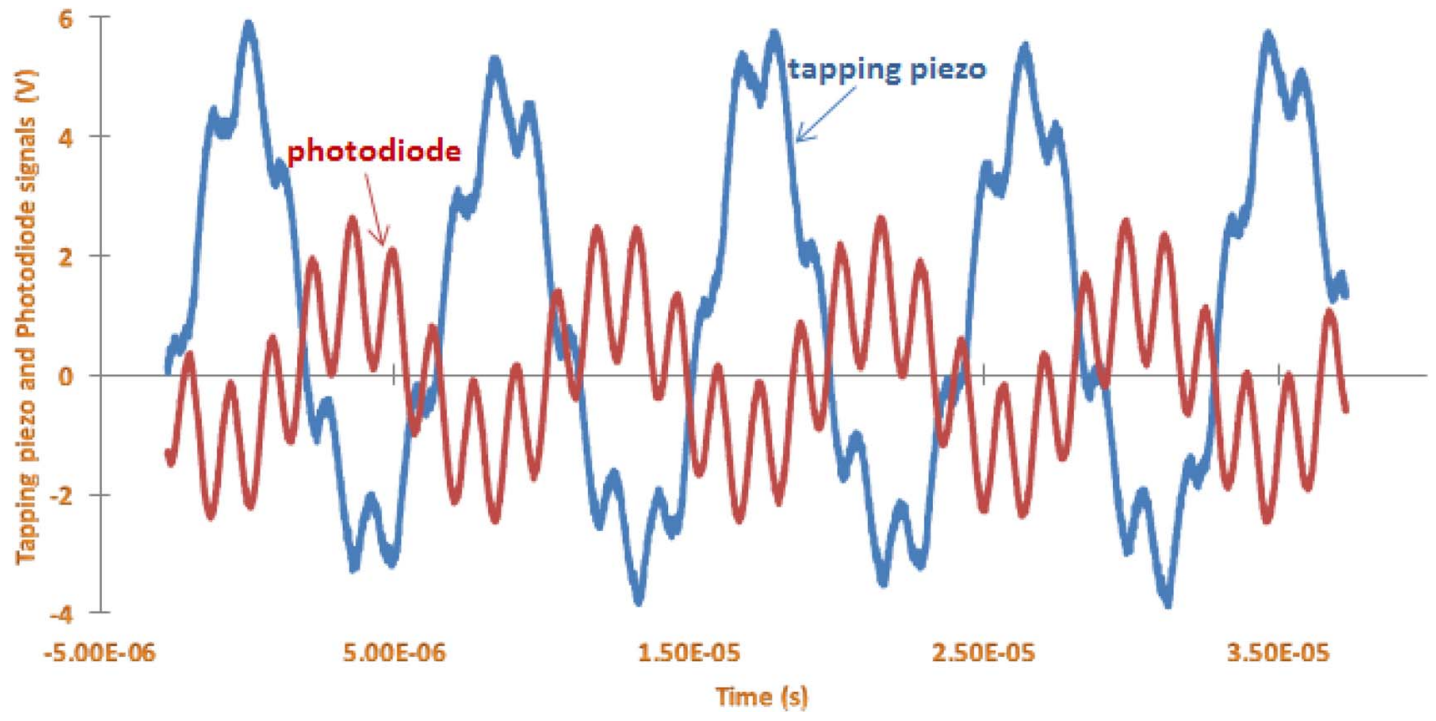

FIG. 2. The tapping piezo and the photodiode signals as a function of time. 

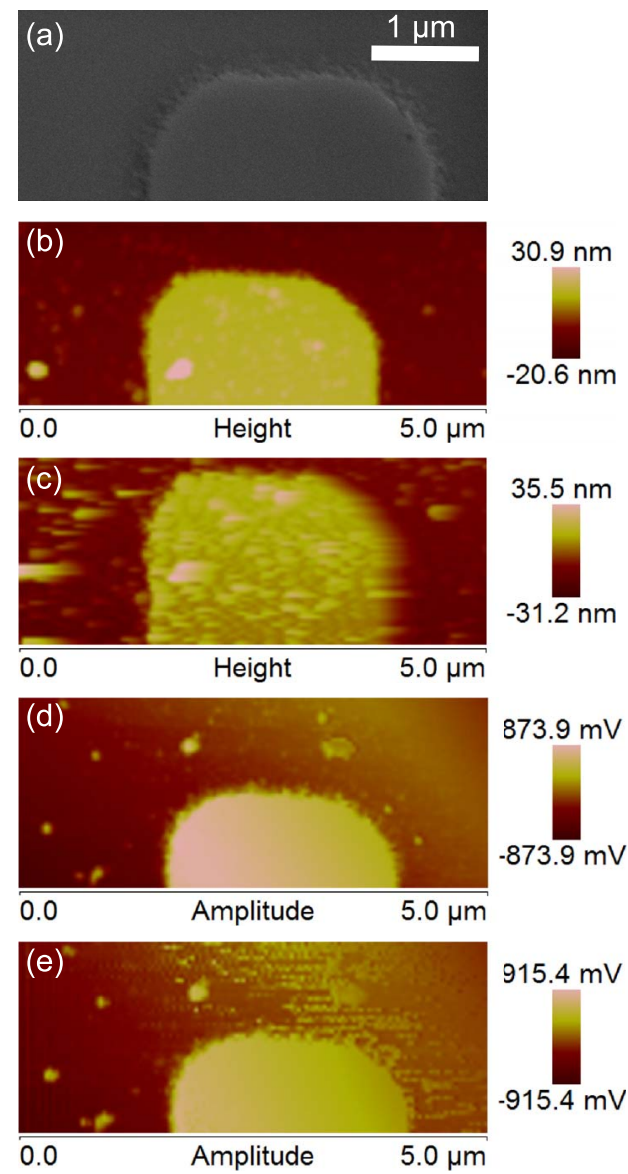

FIG. 3. (a) The SEM micrograph of the sample silicon grating with $25 \mathrm{~nm}$ step height. The topography images obtained by conventional tapping-mode at (b) $1 \mathrm{~Hz}$ and (c) $10 \mathrm{~Hz}$ scan rates. The topography images obtained by fast actuation method at (d) $0.5 \mathrm{~Hz}$ and (e) $10 \mathrm{~Hz}$ scan rates.

approached to the sample. We can also clearly observe the second eigenmode oscillation superimposed on the fundamental eigenmode oscillation in the photodiode signal. Note that the second eigenmode oscillation amplitude looks comparable to the fundamental one since it has a higher sensitivity. The method of laser beam deflection microscopy or finite element simulations can be helpful to visualize the cantilever eigenmode shapes in multifrequency excitation. ${ }^{27}$ In this way, the desired higher flexual eigenmode vibration can be chosen as sensing signal for any type of cantilever. We observe that the image gets distorted at the upper right portion when the scan rate is increased from $0.5 \mathrm{~Hz}$ to $10 \mathrm{~Hz}$ and the amplitude range is changed by approximately $5 \%$.

A major drawback in our imaging experiment was the tipsample engagement process. Since we implemented the additional electronics on a commercial AFM setup, during the engagement process instead of higher eigenmode oscillation, we had to use the fundamental eigenmode oscillation amplitude in the feedback of the internal AFM controller (disabled after engagement). The external PI controller that uses the higher eigenmode oscillation amplitude was also active during the engagement and all the adjustments were done by trimmer potentiometers. Therefore, we could not optimally tune the actuation range, $Q$-control, PI controller, and the set-point parameters in the imaging experiment. Despite all these factors,

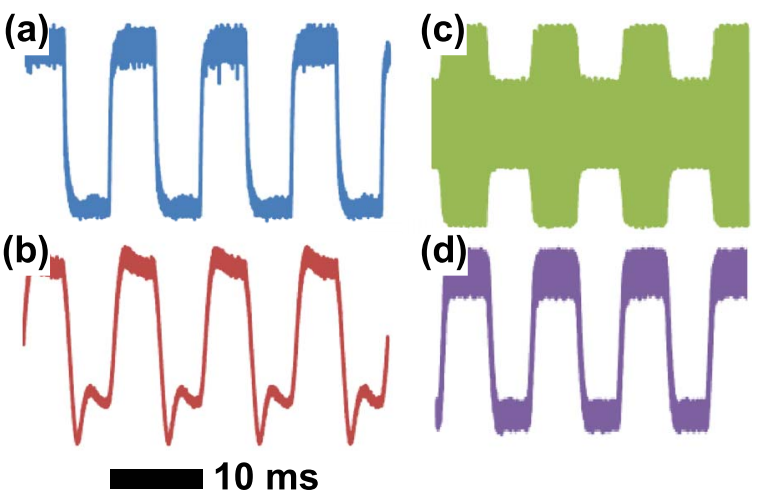

FIG. 4. The effect of tuning in $Q$-control. (a) Modulated second eigenmode oscillation amplitude at $100 \mathrm{~Hz}$. (b) Fundamental eigenmode oscillation amplitude with untuned $Q$-control. (c) The VGA output and (d) the fundamental eigenmode oscillation amplitude with tuned $Q$-control.

we have shown that with the proposed actuation method one can obtain the topography image of a standard sample. In fact, the conventional tapping-mode image gets heavily distorted even at $5 \mathrm{~Hz}$ (data not shown) and the distortion gets even more pronounced at $10 \mathrm{~Hz}$. However, we obtained a relatively better topography image of the same sample at the same rate by the fast actuation method. A possible cause of the distortion at the upper right portion of the image could be the tilt on the sample surface since we could not optimally adjust the actuation range during the engagement process. Actually, setting the internal feedback controller gain to zero may not be ideal as we had to do in the experiment, but instead letting the $\mathrm{Z}$-actuator of the AFM system balance the low-frequency topographic variations and the tilt on the sample surface might be a better approach.

The AFM setup that we used is inherently not suited to high-speed imaging due to its limited detection bandwidth and low scan rate of the X-Y scanner. Therefore to see how fast we can actuate the cantilever with the proposed method, we created a false error signal to mimic a step-like sample surface and we looked at the response of the actuator, i.e., the fundamental eigenmode oscillation amplitude. We modulated the second eigenmode oscillation by $50 \%$ as shown in Fig 4(a), where the cantilever is out of contact with the sample surface. The fundamental eigenmode amplitude in response to the artificial error signal created by the modulation at 100 $\mathrm{Hz}$ is seen in Fig. 4(b) . We see that the response is not perfect because of untuned $Q$-control. After tuning the $Q$-control we obtain the VGA output, i.e., the excitation signal and the fundamental eigenmode oscillation amplitude in response to the same modulation signal in Figs. 4(c) and 4(d). This result tells us that the actuation signal is capable of tracking $100 \mathrm{~Hz}$ step-like error signal and the increase in actuation speed is demonstrated.

We found that the modulation frequency of the artificial error signal can be increased up to $200 \mathrm{~Hz}$ without any significant deterioration in the fundamental eigenmode (actuator) response (Fig. 5). Considering that the error signal is a result of a step-like sample topography then the actuation rate will determine the maximum scan rate. In fact, we simulated a cantilever with a fundamental resonance of $300 \mathrm{kHz}$ on a 


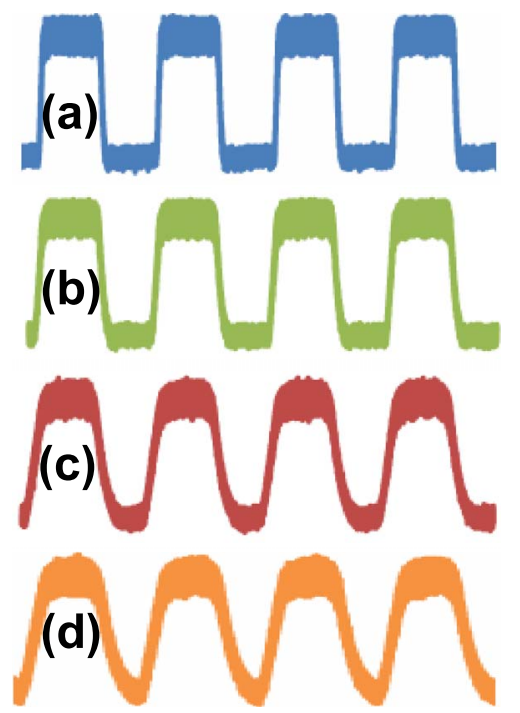

FIG. 5. The response of fundamental eigenmode oscillation amplitude (actuation signal) to the modulated second eigenmode oscillation amplitude (sensing signal) at (a) $50 \mathrm{~Hz}$, (b) $100 \mathrm{~Hz}$, (c) $200 \mathrm{~Hz}$, and (d) $300 \mathrm{~Hz}$.

sample surface with $10 \mathrm{~nm}$ tall step. We found that the minimum required time to image that feature with a specific accuracy is $360 \mu \mathrm{s} .{ }^{19}$ We also found that the imaging bandwidth is almost directly proportional to the cantilever resonance frequency. Given that information we expect that the maximum modulation frequency must be about $(1 / 360 \mu \mathrm{s}) \times(113 \mathrm{kHz} /$ $300 \mathrm{kHz}) \approx 1 \mathrm{kHz}$. This result confirms that our electronics did not function optimally during this particular experiment. Note that we had to select a cantilever such that its second eigenmode resonance must be within the bandwidth of the AFM system. By using small cantilevers that have fundamental resonance frequencies greater than $1 \mathrm{MHz},{ }^{5,6}$ and by integrating a fast $\mathrm{X}-\mathrm{Y}$ scanner ${ }^{15,18}$ into the AFM system, the proposed actuation method can be much effectively used in high-speed dynamic AFM imaging.

\section{CONCLUSION}

In summary, we performed the proof-of-principle experiments of a high-speed actuation method. This method can be utilized in any dynamic AFM system that employs ordinary cantilevers. It was shown theoretically under certain conditions that 24 frames/s imaging speed can be achieved with a cantilever having a resonance frequency of $600 \mathrm{kHz}$ to acquire $100 \times 100$ pixels image in the FM-AFM system. ${ }^{19}$ Hence we expect a faster imaging speeds by using small cantilevers with spring constants less than $1 \mathrm{~N} / \mathrm{m}$ and resonance frequencies greater than $1 \mathrm{MHz}$ as employed in systems with optical beam deflection detection. ${ }^{5,6}$ As the high-speed AFM applications growing rapidly, the presented technique could help to advance the current technology. Ultimately, implementing the method in systems with small cantilevers and fast X-Y scanners can enable the dynamic-AFM systems to be used much effectively in industrial scale nanometrology at high scan rates.

\section{ACKNOWLEDGMENTS}

This work is supported by TUBITAK (Grant No. 110T732)

${ }^{1}$ D. A. Walters, J. P. Cleveland, N. H. Thomson, P. K. Hansma, M. A. Wendman, G. Gurley, and V. Elings, Rev. Sci. Instrum. 67, 3583 (1996).

${ }^{2}$ T. E. Schaffer and P. K. Hansma, J. Appl. Phys. 84, 4661 (1998).

${ }^{3}$ M. B. Viani, T. E. Schaffer, G. T. Paloczi, L. I. Pietrasanta, B. L. Smith, J. B. Thompson, M. Richter, M. Rief, H. E. Gaub, K. W. Plaxco, A. N. Cleland, H. G. Hansma, and P. K. Hansma, Rev. Sci. Instrum. 70, 4300 (1999).

${ }^{4}$ T. Ando, T. Uchihashi, and T. Fukuma, Prog. Surf. Sci. 83, 337 (2008).

${ }^{5}$ G. E. Fantner, G. Schitter, J. H. Kindt, T. Ivanov, K. Ivanova, R. Patel, N. Holten-Andersen, J. Adams, P. J. Thurner, I. W. Rangelow, and P. K. Hansma, Ultramicroscopy 106, 881 (2006).

${ }^{6}$ T. Ando, Nanotechnology 23, 062001 (2012).

${ }^{7}$ S. R. Manalis, S. C. Minne, and C. F. Quate, Appl. Phys. Lett. 68, 871 (1996).

${ }^{8}$ T. Sulchek, S. C. Minne, J. D. Adams, D. A. Fletcher, A. Atalar, C. F. Quate, and D. M. Adderton, Appl. Phys. Lett. 75, 1637 (1999).

${ }^{9}$ T. Sulchek, R. Hsieh, J. D. Adams, S. C. Minne, C. F. Quate, and D. M. Adderton, Rev. Sci. Instrum. 71, 2097 (2000).

${ }^{10}$ T. Akiyama, U. Staufer, and N. F. de Rooij, Rev. Sci. Instrum. 73, 2643 (2002).

${ }^{11}$ B. Rogers, L. Manning, T. Sulchek, and J. D. Adams, Ultramicroscopy 100, 267 (2004).

${ }^{12}$ F. L. Degertekin, A. G. Onaran, M. Balantekin, W. Lee, N. A. Hall, and C. F. Quate, Appl. Phys. Lett. 87, 213109 (2005).

${ }^{13}$ A. G. Onaran, M. Balantekin, W. Lee, W. L. Hughes, B. A. Buchine, R. O. Guldiken, Z. Parlak, C. F. Quate, and F. L. Degertekin, Rev. Sci. Instrum. 77, 023501 (2006).

${ }^{14}$ J. H. Kindt, G. E. Fantner, J. A. Cutroni, and P. K. Hansma, Ultramicroscopy 100, 259 (2004).

${ }^{15}$ A. D. L. Humphris, M. J. Miles, and J. K. Hobbs, Appl. Phys. Lett. 86, 034106 (2005).

${ }^{16}$ T. Fukuma, Y. Okazaki, N. Kodera, T. Uchihashi, and T. Ando, Appl. Phys. Lett. 92, 243119 (2008).

${ }^{17}$ B. Zhao, J. P. Howard-Knight, A. D. L. Humphris, L. Kailas, E. C. Ratcliffe, S. J. Foster, and J. K. Hobbs, Rev. Sci. Instrum. 80, 093707 (2009).

${ }^{18}$ C. Braunsmann and T. E. Schäffer, Nanotechnology 21, 225705 (2010).

${ }^{19}$ M. Balantekin, Ultramicroscopy 149, 45 (2015).

${ }^{20}$ T. Sulchek, R. Hsieh, J. D. Adams, G. G. Yaralioglu, S. C. Minne, C. F. Quate, J. P. Cleveland, A. Atalar, and D. M. Adderton, Appl. Phys. Lett. 76, 1473 (2000).

${ }^{21}$ J. Tamayo, A. D. L. Humphris, and M. J. Miles, Appl. Phys. Lett. 77, 582 (2000).

${ }^{22}$ J. Tamayo, A. D. L. Humphris, R. J. Owen, and M. J. Miles, Biophys. J. 81, 526 (2001).

${ }^{23}$ M. Antognozzi, M. D. Szczelkun, A. D. L. Humphris, and M. J. Miles, Appl. Phys. Lett. 82, 2761 (2003).

${ }^{24}$ J. Tamayo, M. Alvarez, and L. M. Lechuga, Sens. Actuators, B 89, 33 (2003).

${ }^{25}$ N. Kodera, H. Yamashita, and T. Ando, Rev. Sci. Instrum. 76, 053708 (2005).

${ }^{26}$ M. Balantekin and F. L. Degertekin, Ultramicroscopy 111, 1388 (2011).

${ }^{27}$ J. Tamayo, V. Pini, P. Kosaka, N. F. Martinez, O. Ahumada, and M. Calleja, Nanotechnology 23, 315501 (2012). 